\title{
Exosome-mediated microRNA signaling from breast cancer cells is altered by the anti-angiogenesis agent docosahexaenoic acid (DHA)
}

Bethany N. Hannafon', Karla J. Carpenter', William L. Berry ${ }^{2}$, Ralf Janknecht ${ }^{2,4}$, William C. Dooley ${ }^{3,4}$ and Wei-Qun Ding ${ }^{1,4^{*}}$

\begin{abstract}
Background: Docosahexaenoic acid (DHA) is a natural compound with anticancer and anti-angiogenesis activity that is currently under investigation as both a preventative agent and an adjuvant to breast cancer therapy. However, the precise mechanisms of DHA's anticancer activities are unclear. It is understood that the intercommunication between cancer cells and their microenvironment is essential to tumor angiogenesis. Exosomes are extracellular vesicles that are important mediators of intercellular communication and play a role in promoting angiogenesis. However, very little is known about the contribution of breast cancer exosomes to tumor angiogenesis or whether exosomes can mediate DHA's anticancer action.
\end{abstract}

Results: Exosomes were collected from MCF7 and MDA-MB-231 breast cancer cells after treatment with DHA. We observed an increase in exosome secretion and exosome microRNA contents from the DHA-treated cells. The expression of 83 microRNAs in the MCF7 exosomes was altered by DHA ( $>2$-fold). The most abundant exosome microRNAs (let-7a, miR-23b, miR-27a/b, miR-21, let-7, and miR-320b) are known to have anti-cancer and/or anti-angiogenic activity. These microRNAs were also increased by DHA treatment in the exosomes from other breast cancer lines (MDA-MB-231, ZR751 and BT20), but not in exosomes from normal breast cells (MCF10A). When DHA-treated MCF7 cells were co-cultured with or their exosomes were directly applied to endothelial cell cultures, we observed an increase in the expression of these microRNAs in the endothelial cells. Furthermore, overexpression of miR-23b and miR-320b in endothelial cells decreased the expression of their pro-angiogenic target genes (PLAU, AMOTL1, NRP1 and ETS2) and significantly inhibited tube formation by endothelial cells, suggesting that the microRNAs transferred by exosomes mediate DHA's anti-angiogenic action. These effects could be reversed by knockdown of the Rab GTPase, Rab27A, which controls exosome release.

Conclusions: We conclude that DHA alters breast cancer exosome secretion and microRNA contents, which leads to the inhibition of angiogenesis. Our data demonstrate that breast cancer exosome signaling can be targeted to inhibit tumor angiogenesis and provide new insight into DHA's anticancer action, further supporting its use in cancer therapy.

\footnotetext{
* Correspondence: weiqun-ding@ouhsc.edu

'Department of Pathology, University of Oklahoma Health Sciences Center,

Oklahoma City, OK 73104, USA

${ }^{4}$ Peggy and Charles Stephenson Cancer Center, Oklahoma City, OK 73104, USA

Full list of author information is available at the end of the article
}

\section{Biomed Central}

(c) 2015 Hannafon et al. This is an Open Access article distributed under the terms of the Creative Commons Attribution License (http://creativecommons.org/licenses/by/4.0), which permits unrestricted use, distribution, and reproduction in any medium, provided the original work is properly credited. The Creative Commons Public Domain Dedication waiver (http:// creativecommons.org/publicdomain/zero/1.0/) applies to the data made available in this article, unless otherwise stated. 


\section{Background}

Docosahexaenoic acid (DHA, 22:6) is a long-chain omega3 polyunsaturated fatty acid and the main component of dietary fish oil that has many health benefits, including anticancer activity $[1,2]$. The anticancer properties of DHA have been demonstrated both in vitro $[3,4]$ and in vivo [5-7]. Importantly, DHA is cytotoxic to tumor cells, with little or no effects on normal cells [3, 8]. Currently, several clinical trials are evaluating DHA supplementation for breast cancer therapy and management (clinicaltrials.gov). These studies underline the potential value of DHA as both a safe preventative agent and as an adjuvant to therapy.

One of the reported anticancer mechanisms of DHA is the ability to suppress tumor angiogenesis. For example, a DHA-supplemented diet suppresses tumor angiogenesis as measured by microvessel counts in a breast cancer nude mouse model [9] and this observation was confirmed in a murine mammary tumor model also fed a fish oil diet [10]. The anti-angiogenic activity of DHA is also described in a human colon cancer model system [11], a fibrosarcoma implantation model in Fischer 344 rats [12], and in human umbilical cord vein endothelial cells [13]. The cellular mechanisms of how DHA suppresses tumor angiogenesis remain unclear. Traditionally, vascular endothelial growth factor (VEGF), which is secreted from cancer cells in response to hypoxia, is considered the key regulator of tumor angiogenesis and current strategies to inhibit tumor angiogenesis are primarily focused on targeting the VEGF pathway [14]. However, recent studies have demonstrated that other cellular signaling molecules, such as exosomes, also mediate tumor angiogenesis [15-17].

Exosomes are small $(50-100 \mathrm{~nm})$ vesicles that have recently been recognized as important mediators of intercellular communication. They carry lipids, proteins, mRNAs and microRNAs that can be transferred to a recipient cell $[18,19]$. Tumor cells have been shown to secrete exosomes in greater amounts than normal cells [20], thus allowing the transfer of tumor-associated signaling molecules to surrounding cells [21-23]. Importantly, the microRNAs in secreted exosomes can be transferred to a recipient cell where they affect post-transcriptional gene regulation [24]. Cancer cell-derived microRNAs can be transferred via exosomes to endothelial cells where they induce pro-angiogenic effects $[15,16]$. These studies underline the role tumor-derived exosomes can play in the tumor microenvironment and in promoting tumor angiogenesis. However, very little is known about the contents and secretion of breast cancer exosomes or ways to manipulate or reduce their influence on cancer progression. In this study we sought to determine how DHA might alter the secretion and contents of breast cancer exosomes thereby suppressing tumor angiogenesis and progression, which may lead to a better understanding of breast cancer biology and novel strategies targeting intercellular communication for breast cancer therapy.

\section{Results}

DHA increases the small RNA contents of breast cancer exosomes

Human breast cancer MCF7 and MDA-MB-231 cells were grown in cell culture medium supplemented with exosome-depleted serum for 3 days and treated with $100 \mu \mathrm{M}$ DHA for $24 \mathrm{~h}$. Whole exosomes were collected from the conditioned medium of DHA-treated and untreated MCF7 cells. The exosomes were fixed and negatively stained for visualization by electron microscopy. We found exosomes of the typical $\sim 100 \mathrm{~nm}$ diameter in both samples (Fig. 1a), (MDA-MB-231 exosomes not shown). The isolated exosomes had detectable CD63 expression, a well-established marker for exosomes, by immunogold labeling and imaging by electron microscopy (Fig. 1b) and by western blot (Fig. 1c). Total RNA was isolated from the exosomes and a RNA profile was generated. We found that the RNA isolated from the MCF7 exosomes was primarily small RNA of less than 1,000 nt and typically absent of ribosomal RNA as compared to the total cellular RNA (Fig. 1d). These observations are similar to previous reports $[25,26]$. However, in the DHA treated exosome RNA sample we observed an increase in the small RNA content of $<200 \mathrm{nt}$ (see Fig. 1d, lower right panel). These results indicate that DHA increases the small RNA content of breast cancer exosomes.

\section{DHA increases microRNA levels in breast cancer cells and exosomes}

To further determine if DHA increases the small RNA content of breast cancer secreted exosomes, a small RNA library was prepared from equal quantities of total RNA isolated from the DHA-treated and untreated MCF7 exosomes and cells. The resulting cDNA library was sequenced and the reads were mapped onto the human genome (Build 36, Mar 2006) and intersected with known mature microRNAs (mirBase, v13; www.mirbase.org). Overall, DHA treatment increased the number of mature microRNAs detected in the MCF7 cells and exosomes. In the cells, 387 microRNAs were detected (at least 1 read) in control MCF7 cells versus 412 detected in the DHA-treated MCF7 cells (increase of 25 microRNAs). In the exosomes, 196 microRNAs were detected in the control exosomes versus 209 detected in the DHA-treated exosomes (increase of 13 microRNAs) (Additional file 1). These results are consistent with the increase in small RNA content observed in the RNA profile (Fig. 1d). Twentysix cellular microRNAs were changed (1.5-fold or greater) by DHA-treatment (19 up-regulated and 7 down-regulated) (see control cell vs. DHA cell in Additional file 1). Three microRNAs (miR-1246, 204-fold; miR-451, 135-fold; and 


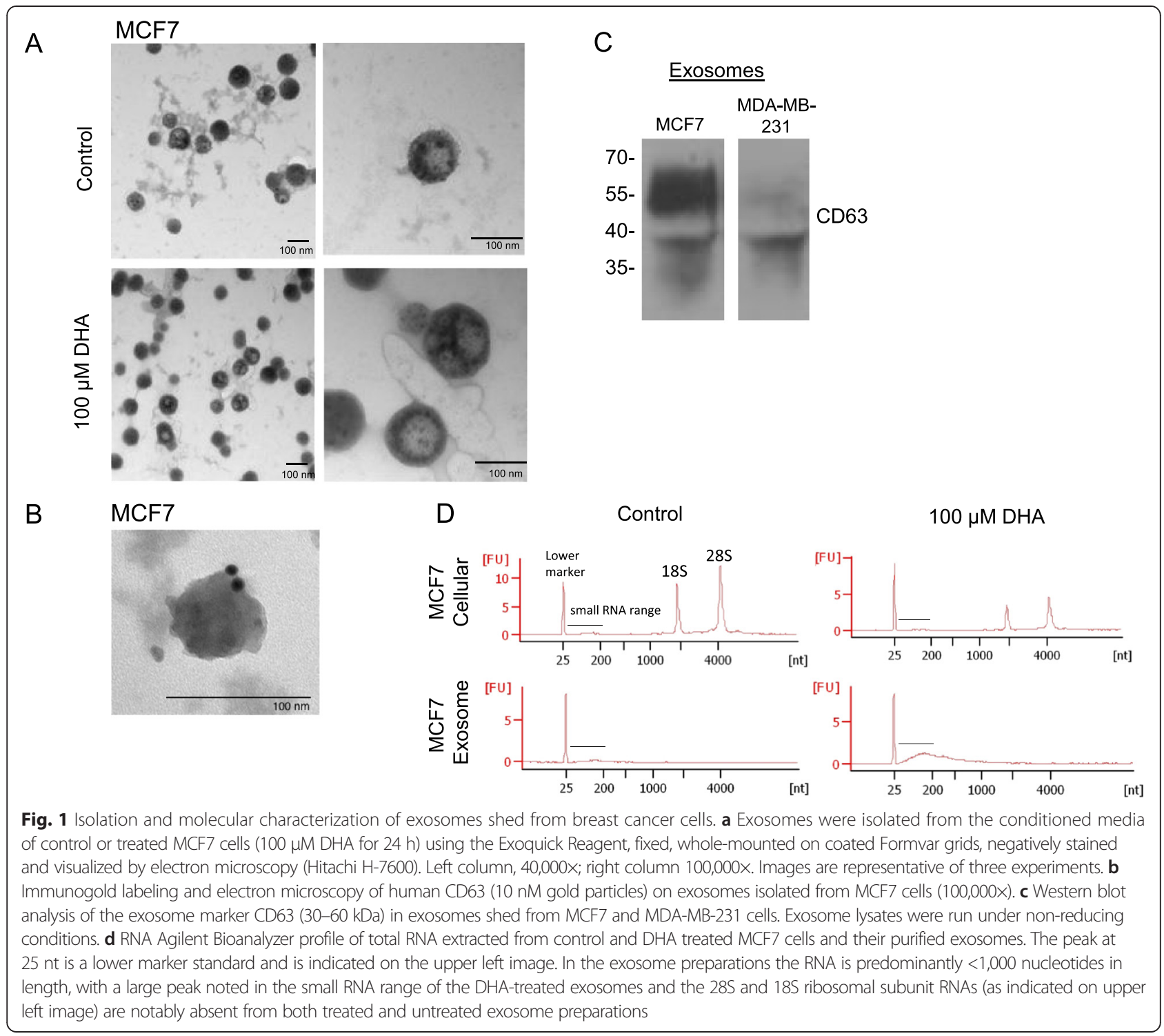

miR-127-3p, 6-fold) were preferentially encapsulated into the exosomes and found at very low levels in the cellular content, which is in agreement with a recent study [27] (see control cell vs. control exosome in Additional file 1). In the DHA-treated exosomes 91 mature microRNAs were detected (at least 1 read), 83 of which were changed by 2 -fold or greater compared to the untreated exosomes and 22 of these microRNAs were present at greater than 1,000 copies in at least one condition; these 22 microRNAs are listed in Table 1. Our results demonstrate that DHA alters the microRNA profile of MCF7 cell-derived exosomes.

DHA increases let-7a, miR-21, miR-23b, miR-27b, and miR-320b levels in exosomes from other breast cancer cell lines, but not from normal breast cells

To determine if the effects of DHA on exosome microRNA levels are specific to only MCF7 breast cancer cells or whether DHA can increase the exosome microRNA levels in normal mammary epithelial cells or other breast cancer types, we cultured a normal mammary epithelial cell line (MCF10A), an ER-positive luminal line (ZR-75-1) and two triple-negative basal-like breast cancer lines (BT20 and MDA-MB-231). Exosomes were isolated from the conditioned media after DHA treatment and total RNA was extracted from the control and DHAtreated exosomes. The expression of 5 of the most abundant microRNAs (let-7a, miR-21, miR-23b, miR-27b, and miR-320b) detected in the DHA-treated MCF7 exosomes was measured in the exosomes from the other breast cell lines by qRT-PCR. As shown in Fig. 2, DHA treatment increased the expression of all 5 of these microRNAs in the DHA-treated exosomes as compared to control exosomes, with the exception of let-7a and miR-21 in MDA-MB-231 cells. In contrast, DHA-treatment did not 
Table 1 DHA increases microRNA expression levels in MCF7 exosomes

\begin{tabular}{|c|c|c|c|c|}
\hline \multirow[t]{2}{*}{ microRNA } & \multicolumn{2}{|c|}{ Mapped reads } & \multirow[t]{2}{*}{ Fold-change } & \multirow[t]{2}{*}{ P-value } \\
\hline & Control & DHA & & \\
\hline miR-23b & 205.5 & 1064.8 & 5.18 & $1.0 \mathrm{E}-20$ \\
\hline miR-378 & 333.3 & 1510.2 & 4.53 & $1.0 \mathrm{E}-20$ \\
\hline let-7f & 2949.3 & 11386.4 & 3.86 & 1.0E-20 \\
\hline miR-21 & 10853.1 & 41786.2 & 3.85 & 1.0E-20 \\
\hline let-7a & 4748.9 & 17764.0 & 3.74 & $1.0 \mathrm{E}-20$ \\
\hline miR-182 & 2044.0 & 6986.1 & 3.42 & $1.0 \mathrm{E}-20$ \\
\hline miR-151-3p & 522.1 & 1760.1 & 3.37 & $1.0 \mathrm{E}-20$ \\
\hline miR-27b & 4082.4 & 13189.9 & 3.23 & 1.0E-20 \\
\hline let-7e & 472.1 & 1521.1 & 3.22 & 1.0E-20 \\
\hline miR-27a & 4032.4 & 12896.6 & 3.20 & 1.0E-20 \\
\hline let-7c & 449.9 & 1434.2 & 3.19 & $1.1 \mathrm{E}-16$ \\
\hline miR-365 & 888.7 & 2824.9 & 3.18 & 1.0E-20 \\
\hline miR-320b & 516.6 & 1553.7 & 3.01 & 1.0E-20 \\
\hline let-7i & 783.2 & 2335.9 & 2.98 & $1.0 \mathrm{E}-20$ \\
\hline miR-30d & 955.3 & 2814.0 & 2.95 & 1.0E-20 \\
\hline miR-26a & 1122.0 & 3302.9 & 2.94 & 1.0E-20 \\
\hline miR-30a & 994.2 & 2890.0 & 2.91 & 1.0E-20 \\
\hline miR-200b & 810.9 & 2314.2 & 2.85 & 1.0E-20 \\
\hline miR-181a & 399.9 & 1086.5 & 2.72 & $7.5 \mathrm{E}-11$ \\
\hline miR-191 & 3204.8 & 7507.6 & 2.34 & $1.0 \mathrm{E}-20$ \\
\hline miR-151-5p & 938.7 & 2151.2 & 2.29 & $1.0 \mathrm{E}-20$ \\
\hline miR-1246 & 4537.9 & 9072.1 & 2.00 & 1.0E-20 \\
\hline
\end{tabular}

The microRNAs with a fold-change $\geq 2$ and mapped reads $\geq 1,000$ in at least one condition are shown. The fold change was calculated by normalizing the reads to the number of mapped reads and the $p$-values were calculated using the Likelihood Ratio Test and adjusted using the Benjamini and Hochberg post-test

increase the expression of these microRNAs in the exosomes from MCF10A cells; rather microRNA expression was decreased by DHA-treatment in these exosomes. These results indicate that the effect of DHA on the microRNA content of their exosomes is specific to cancer cells and is not dependent on the breast cancer subtype.

DHA increases exosome secretion from breast cancer cells To determine if exosome secretion is altered by DHA we measured CD63 expression in DHA-treated and control exosomes secreted from MCF7 and MDA-MB-231 cell lines by western blot. We found increased levels of CD63 protein in the DHA-treated exosome preparations from both cell lines (Fig. 3a). In order to monitor exosome production and measure their release we generated MCF7 and MDA-MB-231 cell lines stably expressing the exosome marker CD63 tagged with GFP (CD63-GFP) (Additional file 2). The MCF7 and MDA-MB-231 CD63GFP cells were grown in cell culture media supplemented with exosome-depleted FBS and treated with $100 \mu \mathrm{M}$ DHA for $24 \mathrm{~h}$. Exosomes were isolated from the conditioned media by ultracentrifugation and suspended in PBS. The presence of GFP in the isolated exosomes was measured by fluorescent spectrometry. We observed a significant increase in exosome secretion from DHA treated versus untreated cell cultures (Fig. 3b), while the cell number was unaffected by DHA treatment (Fig. 3c). These results indicate that DHA increases exosome secretion from breast cancer cell lines.

Breast cancer exosomes are absorbed by endothelial cells and DHA treatment inhibits endothelial cell tube formation without affecting VEGF levels

We then asked whether the anti-angiogenesis activity of DHA could be due to the DHA-induced changes in exosome secretion and microRNA contents that we had observed. First, to determine if breast cancer exosomes can be readily transferred to endothelial cells we cultured the endothelial cell line EA.hy926 on matrigel in the presence of purified exosomes isolated from MCF7 cells that were pre-labeled with the nucleic acid binding dye, acridine orange (AO). The purified AO-labeled exosomes were applied to EA.hy926 cells and imaged by fluorescent microscopy. The presence of AO-exosomes in endothelial cells was observed as early as $2 \mathrm{~h}$ (not shown) and $24 \mathrm{~h}$ (Fig. 3d) indicating that breast cancer exosomes are readily transferred to endothelial cells. Then to determine if tube-formation (in vitro angiogenesis) by endothelial cells is affected by the DHA-induced changes in the breast cancer exosomes, equal volumes of exosomes isolated from control and DHA-treated MCF7 cells were applied to cultures of EA.hy926 plated on matrigel. Tube formation by EA.hy926 cells was significantly inhibited by the exosomes purified from DHA-treated MCF7 cells compared to those purified from control MCF7 cells (Fig. 3e). These results suggest that DHA suppresses tumor angiogenesis via modulating breast cancer cell-derived exosome contents. This conclusion was further supported by the observation that DHA and troglitazone (a PPARY ligand) did not alter VEGF secretion, while clofibrate (a PPAR $\alpha$ ligand) reduced VEGF secretion from MCF7 cells (Fig. 3f).

\section{microRNAs released by DHA-treated breast cancer cells are transferred to endothelial cells}

Several of the most abundant microRNAs in the DHAtreated exosomes have known activity in targeting endothelial cells and suppressing angiogenesis, such as miR-21 [28], miR-23b [29], miR-27b [30] and miR-320b [31]. Therefore, it is highly likely that these microRNAs contribute to the anti-angiogenesis activity of DHA. To test whether these microRNAs can be absorbed by endothelial cells, MCF7 cells were co-cultured with EA.hy926 cells for $24 \mathrm{~h}$ and the 

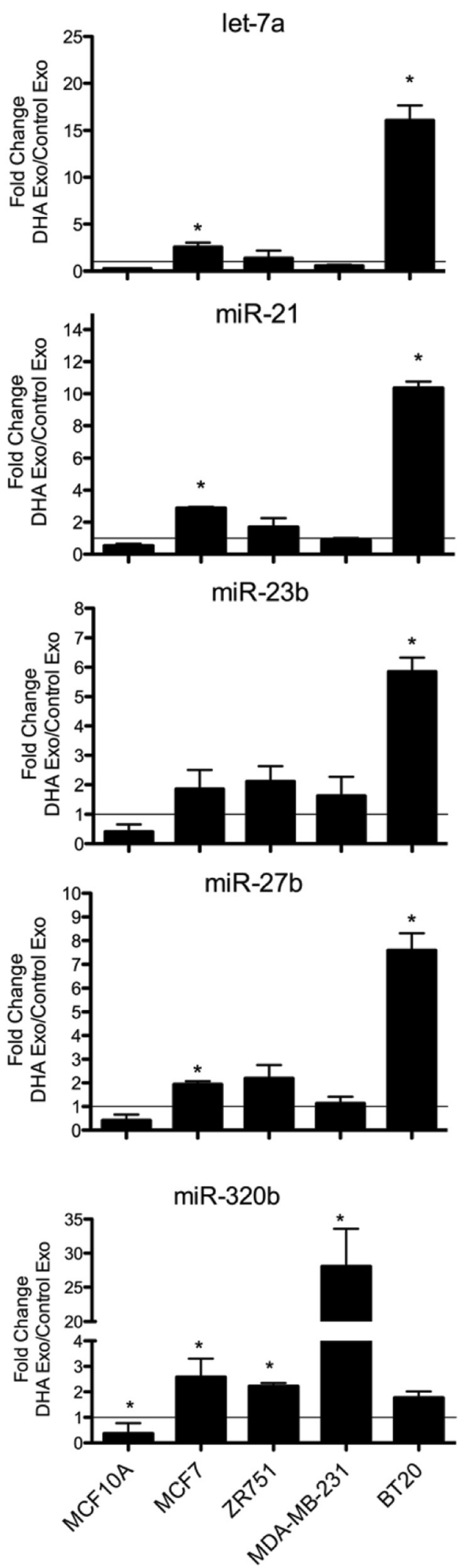

Fig. 2 Validation of DHA-induced microRNA expression changes in exosomes secreted from breast cancer cells. MCF10A, MCF7, ZR751, MDA-MB-231 and BT20 breast cancer cells were plated at a density of $5 \times 10^{6}$ cells per $150 \mathrm{~mm}$ dish and cultured for 3 days. Cells were treated with $100 \mu \mathrm{M}$ DHA for $24 \mathrm{~h}$. Exosomes were collected from the conditioned media by ultracentrifugcation, total RNA was extracted, reverse transcribed and amplified by real-time PCR using microRNA specific primers ( $n=3$, error bars $=\mathrm{SE}$ ). The fold change in expression levels was calculated using the $\Delta \Delta \mathrm{Ct}$ method. ${ }^{*} p<0.05$ by Student's t test

MCF7 cells were treated with DHA. RNA was extracted from the endothelial cells and the expression levels of these microRNAs were measured by qRT-PCR. As shown in Fig. $4 \mathrm{a}$ the expression of let-7a, miR-21, miR-23b, miR-27b, and miR-320b were dramatically increased in the endothelial cells by DHA treatment of the MCF7 cells. A similar microRNA expression pattern was obseved when DHAterated exosomes were directly applied to EA.hy926 cells (Additional file 3). These results indicate that the microRNAs carried by the DHA-treated breast cancer cell exosomes are readily transferred to endothelial cells.

\section{miR-23b, miR-27b, and miR-320b inhibit tube formation} by endothelial cells

To determine which microRNA cargo carried by the MCF7 exosomes might modulate the anti-angiogenesis effects of DHA we transfected EA.hy926 cells with microRNA mimics for miR-21, miR-23b, miR-27b, miR-320b, let-7a or scramble control. microRNA overexpression in the EA.hy926 cells was confirmed with at least a 10-fold increase in expression as determined by qRT-PCR $48 \mathrm{~h}$ post-transfection. Following microRNA mimic transfection the cells were plated on matrigel and cultured. Tube formation by the endothelial cells was quantitated by branchpoint counting (Fig. 4b) and imaged by light microscopy (Fig. 4c), as we have previously described [32]. As shown, increased levels of miR-23b, miR-27b, and miR-320b in the endothelial cells dramatically reduced tube formation by the endothelial cells, whereas overexpression of let-7a and miR-21 did not reduce the tube formation, suggesting that the exosomal microRNAs, miR-23b, miR-27b, and miR320b, mediate DHA's anti-angiogenesis activity.

\section{Co-cultivation of breast cancer cells or microRNA mimic transfection of miR-23b or miR-320b inhibits pro- angiogenesis target mRNA expression}

Several previously defined and validated target genes of miR-23b and miR-320b are known to encode proteins that modulate angiogenesis, including plasminogen activator (PLAU) [33], angiomotin like-1 (AMOTL1) [34], neuropilin 1 (NRP1) [31], and v-ets avian erythroblastosis virus E26 oncogene homolog 2 (ETS2) [35]. We asked whether exosome transfer of miR-23b and miR$320 \mathrm{~b}$ to endothelial cells would affect expression of these 


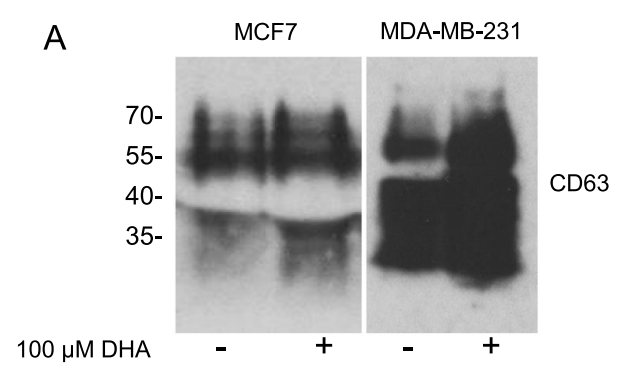

B

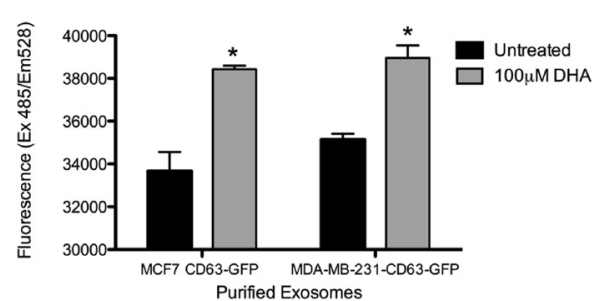

C

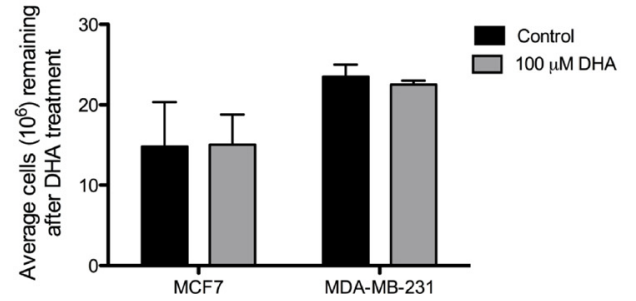

D

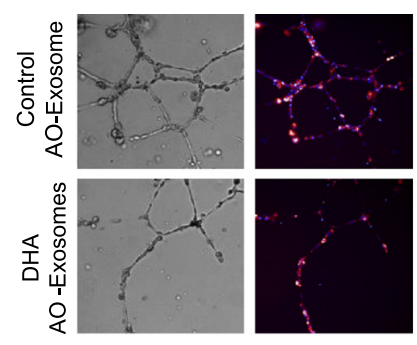

E

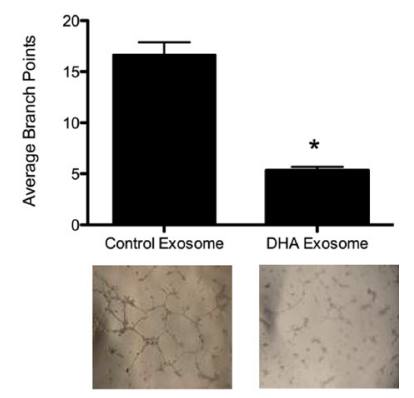

$\mathrm{F}$

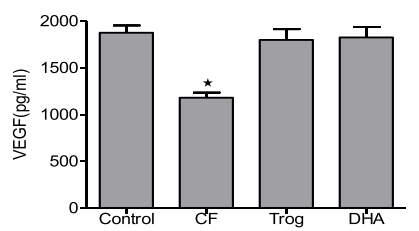

Fig. 3 DHA increases exosome secretion from breast cancer cells and reduces tube formation by endothelial cells without affecting VEGF secretion. a Western blot analysis of CD63 expression in exosomes isolated from MCF7 and MDA-MB-231 cells after treatment with DHA. Equal volumes of exosome protein lysate were loaded and SDS-PAGE was run under non-reducing conditions. b CD63-GFP tagged exosomes secreted into cell culture medium from DHA-treated or untreated MCF7 and MDA-MB-231 breast cancer cells were isolated by ultracentrifugation, re-suspended in PBS and the GFP levels were measured by fluorescent spectrometry, ${ }^{*} p<0.0001$ using one-way ANOVA $(n=3)$. c MCF7 and MDA-MB-231 breast cancer cells were treated with $100 \mu \mathrm{M}$ DHA for $24 \mathrm{~h}$. The media was removed for exosome isolation and the breast cancer cells that remained after DHA treatment were counted and are expressed as the average cell number per $10^{6}$ cells $(n=3$, error bars $=$ SE). $\mathbf{d}$ MCF7 cells were treated with $100 \mu \mathrm{M} \mathrm{DHA}$ for $24 \mathrm{~h}$, the exosomes from the treated and untreated cultures were isolated from the culture media by ultracentrifugation and stained with acridine orange (AO). The AO-labeled exosomes $(100 \mu \mathrm{g})$ were incubated with EA.hy926 cells $\left(1 \times 10^{4}\right)$ seeded on ECMatrix in a 12-well plate for $24 \mathrm{~h}$ and imaged with a Perkin Elmer Operetta at 40x magnification, bright field (left) and fluorescence (right), $460 \mathrm{~nm}$ excitation and $650 \mathrm{~nm}$ emission. e Approximately 1/10 of the exosomes isolated from a confluent $100 \mathrm{~mm}$ plate of DHA-treated or untreated MCF7 cells were mixed with $1 \times 10^{4}$ EA.hy 926 cells and seeded on ECMatrix in a 96-well plate and incubated for $18 \mathrm{~h}$. Tube formation was visualized with a light microscope (lower) and counted (upper) *, $p<0.001$ using one-way ANOVA $(n=4)$. f MCF7 cells were treated with $100 \mu \mathrm{M}$ DHA, $1 \mathrm{mM}$ clofibrate (CF) or $20 \mu \mathrm{M}$ troglitazine (Trog) for $24 \mathrm{~h}$ and VEGF in the medium was analyzed by ELISA and expressed as pg/ml. *, $p<0.001$ using one-way ANOVA $(n=4-6)$

pro-angiogenesis target genes. To address this, we cocultured EA.hy926 cells with MCF7 cells for $24 \mathrm{~h}$. As shown in Fig. 4d, treatment of the co-cultured MCF7 cells with DHA dramatically decreased the expression of PLAU, AMOTL1, NRP1 and ETS2 in the endothelial cells. Direct application of microRNA mimics to the EA.hy926 cells for miR-23b repressed the expression of its target genes PLAU and AMOTL1 (Fig. 4e) and mimics for miR-320b repressed the expression of its target genes NRP1 and ETS2 (Fig. 4f). These results demonstrate that the DHA-induced changes in the microRNA contents of breast cancer secreted exosomes, particularly the increase in miR-23b and miR-320b, can suppress the expression of pro-angiogenesis microRNA target genes.
Rab27A knockdown reverses DHA-induced exosome-mediated microRNA transfer and inhibition of tube formation by endothelial cells

To confirm that the observed effects of DHA on the endothelial cells are dependent on breast cancer exosome secretion and contents, we knocked down the expression of the small Rab GTPase, Rab27A. Rab27A has been shown to control multivesicular endosome membrane docking at the plasma membrane and exosome secretion [36]. The expression of Rab27A was knocked down using a miR-30 based shRNA entry vector with dsRed2 coexpression. The shRab27A-dsRed vector was transduced into MCF7-CD63-GFP and MDA-MB-231-CD63-GFP cells. Expression of the shRab27A construct was confirmed 


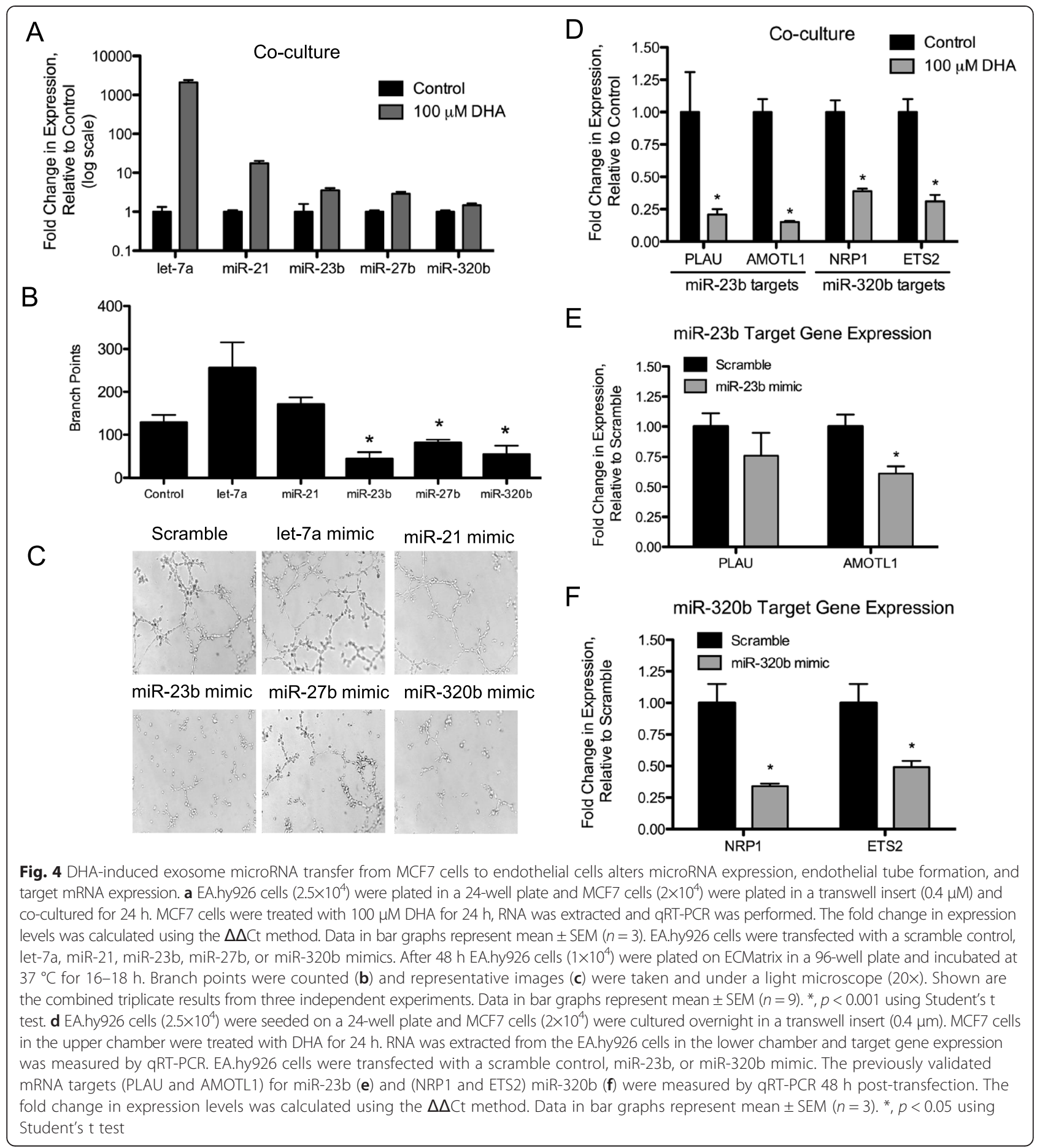

by detection of dsRed levels by fluorescent microscopy (Fig. 5a). Knockdown of the Rab27A protein and mRNA was also confirmed by western blot analysis (Fig. 5b) and qRT-PCR (Fig. 5c), respectively. As shown in Fig. 5d exosome secretion was significantly reduced by Rab27A knockdown. To determine if the effects of DHA on endothelial cell tube formation are dependent on Rab27A-mediated exosome secretion, EA.hy926 cells were seeded on matrigel and co-cultured with the MCF7 CD63-GFP, shRab27AMCF7-CD63, MDA-MB-231 CD63-GFP, or shRab27AMDA-MB-231-CD63 cells plated in a transwell insert. DHA treatment reduced tube formation by the EA.hy 926 cells when co-cultured with the wildtype or CD63-GFP breast cancer cells. However, branch-point formation was increased by DHA-treatment in the shRab27A co-cultures (Fig. 5e), suggesting that the anti-angiogenesis effects of 


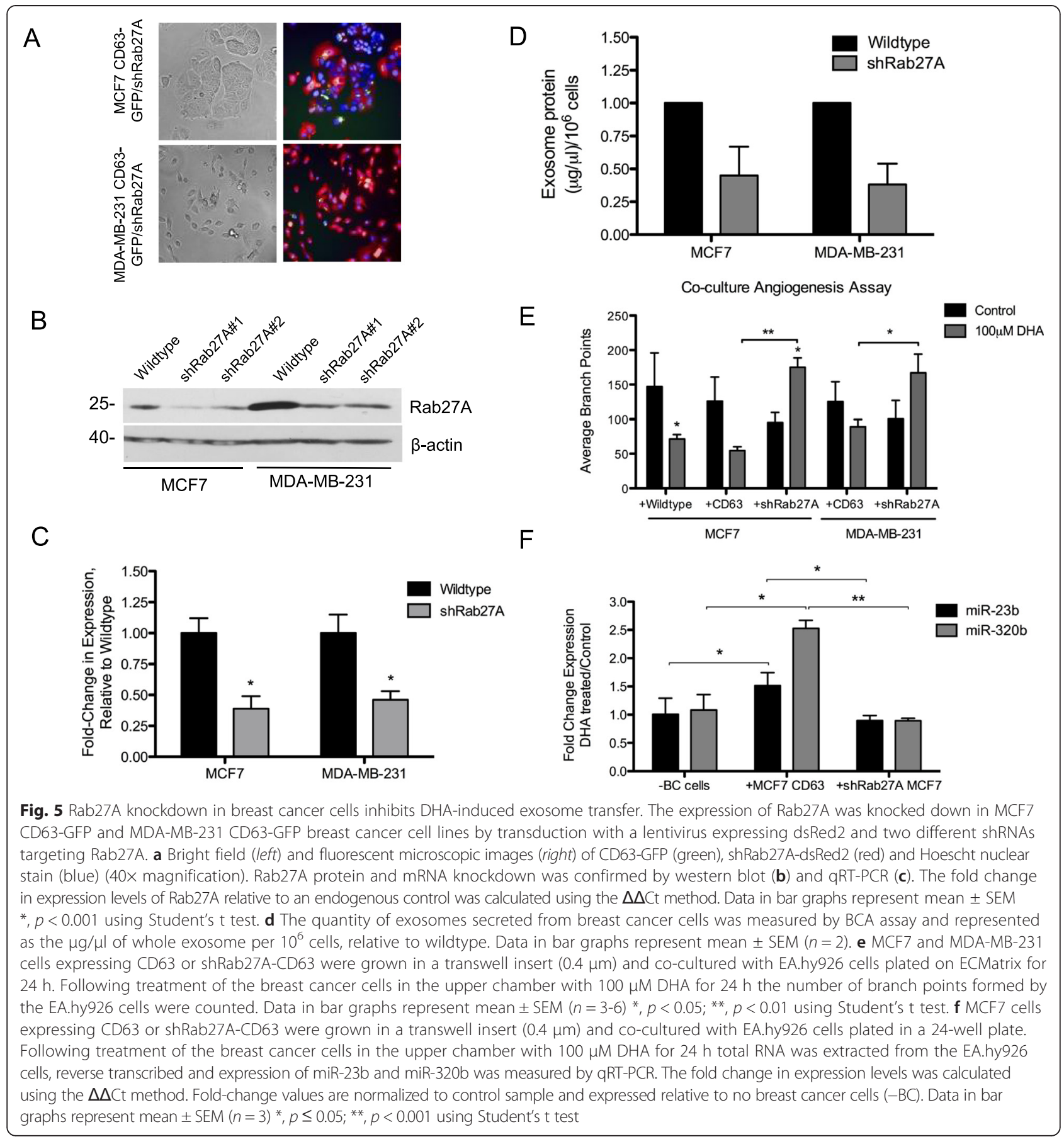

DHA are dependent on Rab27A-mediated exosome secretion. To test whether the transfer of miR-23b or miR-320b occurs with Rab27A knockdown, EA.hy926 cells were plated on the surface of a 24-well plate and cultured alone or co-cultured with MCF7 CD63-GFP or shRab27A MCF7 CD63-GFP cells and treated with DHA for 24 h. As shown in Fig. 5f, the DHA-induced exosome-mediated transfer of miR-23 and miR-320b was inhibited by Rab27A knockdown as compared to CD63-GFP cells. These results suggest that the anti-angiogenesis effects of DHA on endothelial cells are dependent on breast cancer exosome secretion and content transmission.

\section{Discussion}

To our knowledge this is the first demonstration that DHA, a natural anticancer compound, alters breast cancer exosome secretion and microRNA contents, which consequently lead to the suppression of endothelial tube 
formation. In addition to revealing new mechanisms of DHA's anticancer action, these novel findings support the concept that breast cancer exosome signaling can be targeted to inhibit tumor angiogenesis.

The importance of exosomes as mediators of cancer progression has been realized in recent years [37]. However, ways to reduce or change their influence on cancer progression has not been demonstrated. In this study we have shown that DHA can alter the microRNA contents and secretion of breast cancer exosomes. We have provided evidence to show that the microRNA contents of cancer-derived exosomes can be altered, and that these changes can influence their biological activity and intercellular communication. These results provide insight into the future possibility of developing new cancer therapeutic strategies targeting exosome secretion and content transmission.

The anti-angiogenic activity of DHA has been known for some time $[9,10,38]$, however the precise mechanism is elusive. In this study we have shown that the exosomes isolated from DHA-treated breast cancer cells were enriched with microRNAs that target several genes involved in endothelial cell migration, regulation of capillary formation and angiogenesis. More importantly, application of these exosomes to endothelial cells inhibited endothelial tube formation. These results strongly suggest that DHA's anti-angiogenesis effects are at least in part mediated through transfer of exosomes. Because exosomes encapsulate and transfer other biologically active proteins, lipids, and mRNAs, it is also likely that DHA may alter other contents of breast cancer exosomes, which may also mediate the anti-angiogenic effects of DHA.

In the present study we showed that co-culture of endothelial cells with DHA-treated breast cancer cells or application of microRNA mimics significantly inhibited tube formation and repressed the expression of proangiogenesis target genes, including PLAU and AMOTL1 for miR-23b and NRP1 and ETS2 for miR-320b. In support of these findings, a recent study has demonstrated that exosomal transfer of miR-320 from diabetic cardiomyocytes to endothelial cells resulted in inhibition of angiogenesis and reduction in ETS2 expression [39], thus suggesting that exosomal transfer of miR-320 may regulate angiogenesis in other biological model systems.

The mechanism of how DHA might be affecting exosome secretion and changes in exosome microRNA contents is unknown at this time. It is known, however, that exosomes are enriched in the sphingolipid ceramide, whose formation is regulated by neutral sphingomyelinase (nSMase) [40]. It has been demonstrated that exosome secretion can be stimulated by ceramide and inhibited by treatment with the nSMase inhibitor GW4869 [41]. Overexpression of nSMase can increase extracellular levels of microRNAs, whereas treatment with GW4869 microRNA secretion can be reduced [24]. Another study has shown that DHA treatment of breast cancer cells in vitro and through dietary supplementation in vivo induces a 30$40 \%$ increase in nSMase activity and ceramide formation; these effects could also be inhibited by addition of GW4869 [42]. Collectively, these studies indicate that DHA may be involved in regulating exosome secretion and microRNA encapsulation through ceramide formation. However, to date no link has been established connecting DHA, exosomes and microRNA secretion. Here we have provided evidence that DHA can induce exosome secretion and alter the microRNA contents of breast cancer exosomes resulting in changes in their biological function. Whether this effect is mediated through nSMase regulation of ceramide production is yet to be determined.

\section{Conclusions}

In conclusion, this study demonstrates that DHA's antiangiogenesis action is highly likely to be mediated through stimulation of exosome secretion and alteration of exosome microRNA contents in breast cancer model systems. These results provide new insight into DHA's anticancer action and further support the use of DHA as an adjuvant for breast cancer therapy.

\section{Methods}

\section{Cell culture}

The human breast cancer cell lines MCF7, MDA-MB231, BT20 and ZR-75-1 were obtained from the American Type Culture Collection (Manassas, VA). The cells were cultivated in DMEM medium supplemented with exosome-depleted $10 \%$ fetal bovine serum (FBS), $100 \mathrm{IU} /$ $\mathrm{mL}$ penicillin and $100 \mu \mathrm{g} / \mathrm{mL}$ streptomycin (Corning/ Mediatech, Inc. Manassas, VA). Exosome depleted FBS was prepared by pelleting the exosomes by ultracentrifugation at $100,000 \times g$ for $2 \mathrm{~h}$ at $4{ }^{\circ} \mathrm{C}$, the resulting supernatant was filtered through a $0.2 \mu \mathrm{m}$ pore filter and then added to cell culture medium. The EA.hy926 endothelial cell line was kindly provided by Dr. Doris Benbrook (University of Oklahoma Health Sciences Center, Oklahoma City, OK) and was cultivated in F12-K (Corning/Mediatech Inc.) medium supplemented with $10 \%$ fetal bovine serum, $50 \mathrm{mg} / \mathrm{mL}$ Heparin (Sigma-Aldrich, St. Louis, MO), $0.05 \mathrm{mg} / \mathrm{mL}$ Endothelial Cell Growth Supplement (ECGS; Corning/Mediatech Inc.), and $100 \mathrm{IU} / \mathrm{mL}$ penicillin and $100 \mu \mathrm{g} / \mathrm{mL}$ streptomycin (Corning/Mediatech Inc.). Cells were routinely maintained in a humidified chamber at $37{ }^{\circ} \mathrm{C}$ and $5 \% \mathrm{CO}_{2}$.

\section{Preparation and application of DHA}

Docosahexaenoic acid was analytical grade (Sigma-Aldrich) and a stock solution was prepared by dissolving DHA in molecular grade ethanol at $30 \mathrm{mM}$ and stored at $-80{ }^{\circ} \mathrm{C}$ under argon gas. For DHA treatment a $5 \mathrm{mM}$ working 
stock was prepared by dilution with $1.5 \mathrm{mM}$ bovine serum albumin in PBS before adding to cell culture. The final concentration of ethanol in the medium was below $0.5 \%$. Control cells were treated with vehicle buffer.

\section{Exosome isolation}

Exosomes were isolated utilizing a combination of centrifugation and ultracentrifugation according to [43] and filtration or the Exoquick-TC reagent (System Biosciences, Mountain View, CA) following the manufacturer's protocol. For ultracentrifugation isolation, conditioned cell culture media was collected and centrifuged at $10,000 \times g$ for $30 \mathrm{~min}$ at $4{ }^{\circ} \mathrm{C}$, to remove cells and large debris. The supernatant was filtered using a $0.22-\mu \mathrm{m}$ pore filter and the exosomes were pelleted at $100,000 \times g$ for $1 \mathrm{~h}$ at $4{ }^{\circ} \mathrm{C}$. The exosome pellet was washed with $10 \mathrm{ml}$ of 1X PBS and pelleted again by centrifugation at 100,000 $\times g$ for $1 \mathrm{~h}$ at $4{ }^{\circ} \mathrm{C}$. The resulting pellet was either suspended in 1X PBS for whole exosome applications or further processed for RNA or protein extraction. Total exosome RNA was extracted using the TRIzol reagent (Invitrogen/Life Technologies, Carlsbad, CA) following the manufacturer's protocol.

\section{Western blot analysis}

Total exosome protein was prepared by re-suspending the exosomes in RIPA Buffer $(50 \mathrm{mM}$ Tris- $\mathrm{HCl} \mathrm{pH} \mathrm{7.4,}$ $150 \mathrm{mM} \mathrm{NaCl}, 0.5 \%$ sodium deoxycholate, $1 \% \mathrm{NP}-40$, and $0.1 \%$ sodium dodecyl sulfate) containing $1 \mathrm{mM}$ phenlymethylsulfonyl fluoride, $5 \mu \mathrm{g} / \mathrm{ml}$ leupeptin, $2 \mu \mathrm{g} / \mathrm{ml}$ aprotinin, and $1 \mu \mathrm{g} / \mathrm{ml}$ pepstatin A. About $30-40 \mu \mathrm{g}$ of protein from each sample was separated on a $10 \%$ SDSPAGE gel, transferred to a PVDF membrane, and blotted with antibodies against CD63 (Santa Cruz Biotechnology, Santa Cruz, CA), Rab27A (Abnova, Taiwan) or $\beta$-actin (Sigma-Aldrich, St. Louis, MO).

\section{Electron microscopy and immunogold labeling}

Exosomes were fixed in $2 \%$ paraformaldehyde. The fixed sample was absorbed onto formvar coated copper grids for $20 \mathrm{~min}$ in a dry environment. Samples were then fixed in $1 \%$ glutaraldehyde for $5 \mathrm{~min}$. After being rinsed in distilled water samples were stained with uranyl oxalate for $5 \mathrm{~min}$ followed by methyl cellulose uranyl acetate for $10 \mathrm{~min}$ on ice. Excess liquid was wicked off of the grid using filter paper, and grids were stored at room temperature until imaging. For immunogold labeling, exosomes were fixed in $2 \%$ paraformaldehyde. Samples were absorbed onto formvar coated copper grids for $20 \mathrm{~min}$ in a dry environment. Samples were washed with PBS three times. Samples then underwent four washes in $50 \mathrm{mM}$ glycine followed by a 10 min blocking step. Exosomes were incubated with CD63 primary antibody for $30 \mathrm{~min}$, and then samples were washed in washing buffer six times. Samples were incubated in secondary antibody conjugated to $10 \mathrm{nM}$ gold particles for $20 \mathrm{~min}$. Finally, samples were washed in PBS, stabilized with glutaraldehyde, washed in water, and counter stained with uranyl oxalate and methyl cellulose uranyl acetate. Imaging was done on a Hitachi H7600 microscope.

\section{CD63 overexpression and Rab27A knockdown}

To overexpress GFP-tagged CD63 the lentiviral expression vector pCT-CD63-GFP was obtained from System Biosciences, Mountain View, CA. To reduce the expression of Rab27A, shRNAs were cloned into the miR-30 based shRNA entry vector pEN_TRmiRc2 with dsRed2 coexpression. pEN_TRmiRc2 was a gift from Iain Fraser (Addgene plasmid \#25750). The following sequences within the Rab27A 3'UTR were targeted: \#1: CCAGCT CAATGTCTTTGAGTAT and \#2: CGCTCAATGTCTTT GAGTATTA. The resulting fragment was cloned into the pLenti CMV Puro DEST expression vector, a gift from Eric Campeau (Addgene plasmid \#17452), using the Gateway LR Clonase II Enzyme (Invitrogen/Life Technologies, Carlsbad, CA). The lentiviral expression vectors were used to generate VSVg pseudotyped lentivirus. Virus production and infection of cells was performed similarly as before [44]. The 3rd generation packaging plasmids pMD2.G (Addgene plasmid \#12259); pMDL/ RRE g/p (Addgene plasmid \#12251), and pRSV-Rev (Addgene plasmid \#12253) were a gift from Didier Trono. The packaging plasmids were co-transfected with the lentiviral expression vector into human embryonic kidney $293 \mathrm{~T}$ cells using the polyethylenimine (Polysciences, Inc. Warrington, PA) transfection method to produce replication deficient lentivirus. After 48 and $72 \mathrm{~h}$ of transfection, supernatants were pooled, filtered through a $0.45-\mu \mathrm{m}$ MCE membrane and concentrated using polyethylene glycol (Sigma-Aldrich) [45, 46]. MCF7 and MDA-MB-231 were infected with lentivirus in the presence of $8 \mu \mathrm{g} / \mathrm{ml}$ polybrene (Sigma-Aldrich). Approximately $48 \mathrm{~h}$ postinfection cells were selected for gene transfer by treating with $1 \mu \mathrm{g} / \mathrm{ml}$ puromycin (InvivoGen, San Diego, CA).

\section{Fluorescent spectrometry, microscopy and cell imaging}

CD63-GFP (copGFP) levels were measured on BioTek Synergy HT plate reader (BioTek Instruments, Inc. Winooski, VT) and detected by excitation at $485 \mathrm{~nm}$ and emission at $528 \mathrm{~nm}$. Endothelial cells were analyzed by fluorescence microscopy using the Operetta High Content Imaging System (PerkinElmer, Waltham, MA). Endothelial cells were plated on Matrigel in Cell Carrier-96 plate from (PerkinElmer) at a density of 10,000 cells per well. Whole exosomes were labeled by combining $1 \mathrm{~mL}$ of whole exosomes isolated by ultracentrifugation with $20 \mu \mathrm{M}$ acridine orange (AO, Molecular Probes/Life Technologies). These exosomes were incubated in the 
dark at room temperature for $1 \mathrm{~h}$, diluted in $20 \mathrm{~mL}$ of PBS, and ultracentrifuged at $100,000 \times g$ for $1 \mathrm{~h}$. The exosome pellet was then resuspended in PBS. AO was detected by excitation at $460 \mathrm{~nm}$, emission at $650 \mathrm{~nm}$ for red fluorescence.

\section{Endothelial tube formation assay (in vitro angiogenesis)}

Endothelial tube formation was measured utilizing the in vitro Angiogenesis Assay Kit (Millipore, Billerica, MA) as we have previously described [32]. EA.hy926 $\left(1 \times 10^{4}\right)$ cells were resuspended in EBM-2 (Lonza Group, Walkersville, $\mathrm{MD}$ ) medium and plated onto the polymerized extracellular matrix. The formation of endothelial tubes was observed microscopically and photographed after $17-24 \mathrm{~h}$ incubation. Tube formation was quantified by branch point counting as specified by the manufacturer.

\section{VEGF secretion}

Secretion of VEGF from MCF-7 cells was determined using an ELISA kit as previously reported [32]. Cells were seeded into 6-well plates at a density of $1 \times 10^{6}$ cells/well and treated with clofibrate or troglitazone for $4 \mathrm{~h}$ prior to placement into the hypoxia chamber for $16 \mathrm{~h}$. The culture medium was then collected, and the level of VEGF in the medium was analyzed following the manufacturer's instructions.

\section{RNA extraction}

Total RNA was extracted using the TRIzol reagent (Invitrogen/Life Technologies) following the manufacturer's protocol. RNA concentration was quantitated using the NanoDrop ND-100 Spectrophotometer (NanoDrop Technologies, Wilmington, DE) and the quality was assessed using the Agilent 2100 Bioanalyzer (Agilent Technologies, Palo Alto, CA), according to the manufacturer's protocol.

\section{Small RNA library preparation and next generation sequencing}

A small RNA library was prepared from $100 \mathrm{ng}$ of total RNA using the TruSeq Small RNA Preparation Kit (Illumina, San Diego, CA). The MiSeq next generation sequencer (Illumina) was used to sequence the resulting cDNA ( $2 x 25 \mathrm{bp}, 50$ cycles). The reads were mapped onto the human genome (hg18 build [47]) and intersected with microRNAs (mirBase.org [48]) using GeneSifter Software (PerkinElmer, Santa Clara CA). Reads were normalized to the mapped reads and significant differences in reads were determined using the Likelihood Ratio Test and the Benjamini and Hochberg post-test. A $p<0.05$ is considered significant. P-values close to 0 are represented as 1.0E-20. The RNA sequencing data discussed in this publication have been deposited in NCBI's Gene Expression Omnibus [49] and are accessible through GEO Series accession number GSE70432 (http://www.ncbi.nlm.nih.gov/geo/query/acc.cgi?acc=GSE70432).

\section{Quantitative real-time reverse transcription PCR}

For microRNA expression analysis complementary DNA from $5 \mathrm{ng}$ of total RNA was synthesized by the addition of a microRNA specific 5X reverse transcription stem-loop primer and the TaqMan microRNA Reverse Transcription Kit, according to the manufacturer's instructions. RealTime PCR was performed by diluting the cDNA product in 2X TaqMan Universal Master Mix II (with UNG) and 20X TaqMan microRNA Expression Assay for each mature microRNA to be measured: miR-21 (ID:000397), miR-23b (ID:00400), miR-27b (ID:000409), miR-320b (ID:002844), and let-7a (ID:000377). All reagents and primers were from Life Technologies. The small ribonuclear RNA RNU6B (ID:001093) served as a microRNA expression normalization control for cellular microRNA expression analysis. However, we found RNU6B to be unreliable for exosome microRNA expression normalization, therefore we used a synthetic Caenorhabditis elegans miR-54 (celmiR-54) RNA oligonucleotide (Integrated DNA Technologies, Coralville, IA) as a spike-in control. Cel-miR-54 has previously been shown not to affect human microRNA detection [50]. The cel-miR-54 ( $0.25 \mathrm{nM})$ oligonucleotide was spiked into each RNA sample prior to complementary DNA synthesis and Real-Time PCR was performed using the TaqMan microRNA assay (ID:001361, Life Technologies). microRNA target gene expression was measured by generating cDNA from $200 \mathrm{ng}$ of total RNA using the iScript cDNA Synthesis kit (Bio-Rad, Hercules, CA). The synthesized cDNA was diluted in 2X iTaq Universal SYBR Green Supermix (Bio-Rad, Hercules, CA) and combined with $4 \mathrm{uM}$ of each forward and reverse primer. Specific primer sequences used are as follows NRP1, forward 5'-CCT TCTGCCACTGGGAACAT-3' and reverse 5' -TTGCCAT CTCCTGTGTGATCC-3'; AMOTL1, forward 5'-CGAGG GACTGAACTAGCCAT-3' and reverse 5'-AGGGGACCC TTTCACCG-3'; PRDX1, forward 5' -CTGGTTGAACCCC AAGCTGATA-3' and reverse 5'-CAGCTGTGGCTTTG AAGTTGG-3'; PLAU, forward 5'-CGACTCCAAAGGCA GCAATGA-3' and reverse 5'-TGGACACACATGTTCCT CCATT' 3 '; ETS2, forward 5'-CTCACCAACAATTCTGG GACTC-3' and reverse 5'-CACATCCAGCAAGGACGA CT-3'; and the normalization control 36B4, forward 5'-AT CAACGGGTACAAACGAGTCCTG-3' and reverse 5' - A AGGCAGATGGATCAGCCAAGAAG-3'. PCR reactions were run on the Bio-Rad CFX 96 Real-Time PCR (Bio-Rad, Hercules, $\mathrm{CA}$ ) instrument under the following conditions: hold at $95{ }^{\circ} \mathrm{C}$ for $10 \mathrm{~min}$, then 40 cycles of $95{ }^{\circ} \mathrm{C}$ for $15 \mathrm{~s}$ and $60{ }^{\circ} \mathrm{C}$ for $1 \mathrm{~min}$. Relative gene expression was assessed using the differences in normalized $\mathrm{Ct}(\Delta \Delta \mathrm{Ct}$ method) after normalization to RNU6B (cellular microRNA) or cel-miR- 
54 (exosome microRNA). Fold changes were calculated using $2^{-\Delta \Delta \mathrm{Ct}}$.

\section{microRNA mimic transfection}

miRIDIAN microRNA mimics (50 $\mathrm{nM}$ ) were transfected into endothelial cells using the DharmaFect Duo reagent (Dharmacon/GE Healthcare, Lafayette, CO) according to manufacturer's protocol.

\section{Statistical analysis}

All statistical analyses were completed using GraphPad Prism software (GraphPad Software, Inc. La Jolla, CA). When appropriate a Student's t-test or One-way analysis of variance (ANOVA) with Dunnett's post-test was used to determine statistically significant differences among control and experimental groups, with a $p<0.05$ or lower as the level of significance.

\section{Additional files}

Additional file 1: Next generation small RNA sequencing data. This Excel file contains the microRNA reads and pairwise analysis results for the control and DHA treated MCF7 cells and exosomes.

Additional file 2: CD63-GFP expression in breast cancer cells. This file contains microscopic images confirming CD63-GFP expression in breast cancer cells.

Additional file 3: Transfer of exosome microRNA from MCF7 exosomes to endothelial cells. This file contains microRNA expression data confirming exosome microRNA transfer from MCF7 exosomes to endothelial cells.

\section{Competing interests}

The authors declare that they have no competing interests.

\section{Authors' contributions}

BNH designed the study, carried out and/or supervised the experiments and drafted the manuscript. KJC conducted the microRNA mimic transfections, and a sub-set of the co-culture and angiogenesis assays. WLB carried out the lentivirus packaging and transduction. RJ participated in the coordination of the study and helped to draft the manuscript. WCD participated in the design of the study. WQD conceived of the study, and participated in its design and coordination and helped to draft the manuscript. All authors read and approved the final manuscript.

\section{Acknowledgements}

This study was supported in part by grants from the American Cancer Society (CNE-117557); the Susan G. Komen for the Cure Foundation (KG081083); the NIH OK-INBRE program (3P20RR016478-09S2); and the Oklahoma Center for the Advancement of Science and Technology (HR14-147). We would like to acknowledge the services and support provided by the Functional Genomics Core facility at the Peggy and Charles Stephenson Cancer Center, the DNA Sequencing and Genomics facility in the Laboratory for Molecular Biology and Cytometry Research at the University of Oklahoma Health Sciences Center for providing the Illumina MiSeq small RNA library construction, sequencing and analysis services, and the Imaging Core Facility at the Oklahoma Medical Research Foundation for electron microscopy image acquisition.

\section{Author details}

'Department of Pathology, University of Oklahoma Health Sciences Center, Oklahoma City, OK 73104, USA. ${ }^{2}$ Department of Cell Biology, University of Oklahoma Health Sciences Center, Oklahoma City, OK 73104, USA. ${ }^{3}$ Department of Surgery, University of Oklahoma Health Sciences Center, Oklahoma City, OK 73104, USA. ${ }^{4}$ Peggy and Charles Stephenson Cancer Center, Oklahoma City, OK 73104, USA.
Received: 12 January 2015 Accepted: 17 June 2015

Published online: 16 July 2015

\section{References}

1. Vaughan VC, Hassing MR, Lewandowski PA. Marine polyunsaturated fatty acids and cancer therapy. Br J Cancer. 2013;108:486-92.

2. Merendino N, Costantini L, Manzi L, Molinari R, D'Eliseo D, Velotti F. Dietary omega -3 polyunsaturated fatty acid DHA: a potential adjuvant in the treatment of cancer. Biomed Res Int. 2013;2013:310186.

3. Chajes V, Sattler W, Stranzl A, Kostner GM. Influence of $n-3$ fatty acids on the growth of human breast cancer cells in vitro: relationship to peroxides and vitamin-E. Breast Cancer Res Treat. 1995;34:199-212.

4. Schley PD, Jijon HB, Robinson LE, Field CJ. Mechanisms of omega-3 fatty acid-induced growth inhibition in MDA-MB-231 human breast cancer cells. Breast Cancer Res Treat. 2005:92:187-95.

5. Kang KS, Wang P, Yamabe N, Fukui M, Jay T, Zhu BT. Docosahexaenoic acid induces apoptosis in MCF-7 cells in vitro and in vivo via reactive oxygen species formation and caspase 8 activation. PLoS One. 2010;5, e10296.

6. Xue M, Wang Q, Zhao J, Dong L, Ge Y, Hou L, et al. Docosahexaenoic acid inhibited the Wnt/beta-catenin pathway and suppressed breast cancer cells in vitro and in vivo. J Nutr Biochem. 2014;25:104-10.

7. Rose DP, Connolly JM, Rayburn J, Coleman M. Influence of diets containing eicosapentaenoic or docosahexaenoic acid on growth and metastasis of breast cancer cells in nude mice. J Natl Cancer Inst. 1995;87:587-92.

8. Grammatikos SI, Subbaiah PV, Victor TA, Miller WM. n-3 and n-6 fatty acid processing and growth effects in neoplastic and non-cancerous human mammary epithelial cell lines. Br J Cancer. 1994;70:219-27.

9. Rose DP, Connolly JM. Antiangiogenicity of docosahexaenoic acid and its role in the suppression of breast cancer cell growth in nude mice. Int J Oncol. 1999:15:1011-5.

10. Mukutmoni-Norris M, Hubbard NE, Erickson KL. Modulation of murine mammary tumor vasculature by dietary n-3 fatty acids in fish oil. Cancer Lett. 2000;150:101-9.

11. Calviello G, Di Nicuolo F, Gragnoli S, Piccioni E, Serini S, Maggiano N, ringali G, Navarra P, Ranelletti FO, Palozza P. n-3 PUFAs reduce VEGF expression in human colon cancer cells modulating the COX-2/PGE2 induced ERK-1 and -2 and HIF-1alpha induction pathway. Carcinogenesis. 2004;25:2303-10.

12. Tevar R, Jho DH, Babcock T, Helton WS, Espat NJ. Omega-3 fatty acid supplementation reduces tumor growth and vascular endothelial growth factor expression in a model of progressive non-metastasizing malignancy. JPEN J Parenter Enteral Nutr. 2002;26:285-9.

13. Tsushima T, Matsubara K, Ohkubo T, Inoue Y, Takahashi K. Docosahexaenoicand eicosapentaenoic acid-bound lysophospholipids are more effective in suppressing angiogenesis than conjugated docosahexaenoic acid. J Oleo Sci. 2012;61:427-32.

14. Kerbel R, Folkman J. Clinical translation of angiogenesis inhibitors. Nat Rev Cancer. 2002;2:727-39.

15. Umezu T, Ohyashiki K, Kuroda M, Ohyashiki JH. Leukemia cell to endothelial cell communication via exosomal miRNAs. Oncogene. 2013;32:2747-2755.

16. Zhuang G, Wu X, Jiang Z, Kasman I, Yao J, Guan Y, Oeh J, Modrusan Z, Bais C, Sampath D, Ferrara N. Tumour-secreted miR-9 promotes endothelial cell migration and angiogenesis by activating the JAK-STAT pathway. EMBO $\mathrm{J}$. 2012;31:3513-23

17. Umezu T, Ohyashiki K, Kuroda M, Ohyashiki JH. Leukemia cell to endothelial cell communication via exosomal miRNAs. Oncogene. 2013;32:2747-55.

18. Henderson MC, Azorsa DO. The genomic and proteomic content of cancer cell-derived exosomes. Front Oncol. 2012;2:38.

19. Tian $T$, Wang $Y$, Wang $H$, Zhu Z, Xiao Z. Visualizing of the cellular uptake and intracellular trafficking of exosomes by live-cell microscopy. J Cell Biochem. 2010;111:488-96.

20. Taylor DD, Gercel-Taylor C. MicroRNA signatures of tumor-derived exosomes as diagnostic biomarkers of ovarian cancer. Gynecol Oncol. 2008;110:13-21.

21. Staubach S, Razawi H, Hanisch FG. Proteomics of MUC1-containing lipid rafts from plasma membranes and exosomes of human breast carcinoma cells MCF-7. Proteomics. 2009;9:2820-35.

22. Ciravolo V, Huber V, Ghedini GC, Venturelli E, Bianchi F, Campiglio M, Morelli D, Villa A, Della Mina P, Menard S et al. Potential role of HER2-overexpressing exosomes in countering trastuzumab-based therapy. J Cell Physiol. 2012;227:658-67.

23. Higginbotham JN, Demory Beckler M, Gephart JD, Franklin JL, Bogatcheva G, Kremers GJ, Piston DW, Ayers GD, McConnell RE, Tyska MJ, Coffey RJ. Amphiregulin exosomes increase cancer cell invasion. Curr Biol. 2011;21:779-86 
24. Kosaka N, Iguchi H, Yoshioka Y, Takeshita F, Matsuki Y, Ochiya T. Secretory mechanisms and intercellular transfer of microRNAs in living cells. J Biol Chem. 2010;285:17442-52.

25. Rosell R, Wei J, Taron M. Circulating microRNA signatures of tumor-derived exosomes for early diagnosis of non-small-cell lung cancer. Clin Lung Cancer. 2009;10:8-9.

26. Kruger S, Abd Elmageed ZY, Hawke DH, Worner PM, Jansen DA, Abdel-Mageed AB, Alt EU, Izadpanah R. Molecular characterization of exosome-like vesicles from breast cancer cells. BMC Cancer. 2014;14:44

27. Pigati L, Yaddanapudi SC, lyengar R, Kim DJ, Hearn SA, Danforth D, Hastings ML, Duelli DM. Selective release of microRNA species from normal and malignant mammary epithelial cells. PLoS One. 2010;5, e13515.

28. Sabatel C, Malvaux L, Bovy N, Deroanne C, Lambert V, Gonzalez ML, Colige A, Rakic JM, Noel A, Martial JA, Struman I. MicroRNA-21 exhibits antiangiogenic function by targeting RhoB expression in endothelial cells. PLoS One. 2011;6, e16979.

29. Wang KC, Garmire LX, Young A, Nguyen P, Trinh A, Subramaniam S, Wang $\mathrm{N}$, Shyy JY, Li YS, Chien S. Role of microRNA-23b in flow-regulation of Rb phosphorylation and endothelial cell growth. Proc Natl Acad Sci U S A. 2010;107:3234-9.

30. Ye J, Wu X, Wu D, Wu P, Ni C, Zhang Z, Chen Z, Qiu F, Xu J, Huang J. miRNA-27b targets vascular endothelial growth factor $C$ to inhibit tumor progression and angiogenesis in colorectal cancer. PLoS One. 2013;8, e60687.

31. Wu YY, Chen YL, Jao YC, Hsieh IS, Chang KC, Hong TM. miR-320 regulates tumor angiogenesis driven by vascular endothelial cells in oral cancer by silencing neuropilin 1. Angiogenesis. 2014;17:247-60.

32. Zhou J, Zhang S, Xue J, Avery J, Wu J, Lind SE, Ding WQ. Activation of peroxisome proliferator-activated receptor alpha (PPARalpha) suppresses hypoxia-inducible factor-1alpha (HIF-1alpha) signaling in cancer cells. J Biol Chem. 2012;287:35161-9.

33. Pellegrino L, Stebbing J, Braga VM, Frampton AE, Jacob J, Buluwela $L$, Jiao LR, Periyasamy M, Madsen CD, Caley MP et al. miR-23b regulates cytoskeletal remodeling, motility and metastasis by directly targeting multiple transcripts. Nucleic Acids Res. 2013:41:5400-12.

34. Helwak A, Kudla G, Dudnakova T, Tollervey D. Mapping the human miRNA interactome by CLASH reveals frequent noncanonical binding. Cell. 2013;153:654-65

35. Wallace JA, Li F, Balakrishnan S, Cantemir-Stone CZ, Pecot T, Martin C, Kladney RD, Sharma SM, Trimboli AJ, Fernandez SA et al. Ets2 in tumor fibroblasts promotes angiogenesis in breast cancer. PLoS One. 2013;8, e71533.

36. Ostrowski M, Carmo NB, Krumeich S, Fanget I, Raposo G, Savina A, F, Schauer K, Hume AN, Freitas RP et al. Rab27a and Rab27b control different steps of the exosome secretion pathway. Nat Cell Biol. 2010;12(19-30):11-3.

37. Hannafon BN, Ding WQ. Intercellular communication by exosome-derived microRNAs in cancer. Int J Mol Sci. 2013;14:14240-69.

38. Spencer L, Mann C, Metcalfe M, Webb M, Pollard C, Spencer D, Berry D, Steward W, Dennison A. The effect of omega-3 FAs on tumour angiogenesis and their therapeutic potential. Eur J Cancer. 2009;45:2077-86.

39. Wang X, Huang W, Liu G, Cai W, Millard RW, Wang Y, Chang J, Peng T, Fan GC. Cardiomyocytes mediate anti-angiogenesis in type 2 diabetic rats through the exosomal transfer of miR-320 into endothelial cells. J Mol Cell Cardiol. 2014;74:139-50.

40. Clarke CJ, Snook CF, Tani M, Matmati N, Marchesini N, Hannun YA. The extended family of neutral sphingomyelinases. Biochemistry. 2006:45:11247-56.

41. Trajkovic K, Hsu C, Chiantia S, Rajendran L, Wenzel D, Wieland F, Schwille P, Brugger B, Simons M. Ceramide triggers budding of exosome vesicles into multivesicular endosomes. Science. 2008;319:1244-7.

42. Wu M, Harvey KA, Ruzmetov N, Welch ZR, Sech L, Jackson K, Stillwell W, Zaloga GP, Siddiqui RA. Omega-3 polyunsaturated fatty acids attenuate breast cancer growth through activation of a neutral sphingomyelinase-mediated pathway. Int J Cancer. 2005;117:340-8.

43. Thery C, Amigorena S, Raposo G, Clayton A. Isolation and characterization of exosomes from cell culture supernatants and biological fluids. Curr Protoc Cell Biol. 2006; Chapter 3:Unit 322

44. Berry WL, Kim TD, Janknecht R. Stimulation of beta-catenin and colon cancer cell growth by the KDM4B histone demethylase. Int J Oncol. 2014:44:1341-8.

45. Marino M, Luce M, Reiser J. Small- to Large-Scale Production of Lentivirus Vectors. In Federico M, editor. Lentivirus Gene Engineering Protocols. Humana Press; 2003. Volume 229. p. 43-55.

46. Marino MP, Luce MJ, Reiser J. Small- to large-scale production of lentivirus vectors. Methods Mol Biol. 2003;229:43-55.
47. Meyer LR, Zweig AS, Hinrichs AS, Karolchik D, Kuhn RM, Wong M, Sloan CA, Rosenbloom KR, Roe G, Rhead B, et al. The UCSC Genome Browser database: extensions and updates 2013. Nucleic Acids Res. 2013;41:D64-9.

48. Kozomara A, Griffiths-Jones S. miRBase: integrating microRNA annotation and deep-sequencing data. Nucleic Acids Res. 2011;39:D152-7.

49. Barrett T, Wilhite SE, Ledoux P, Evangelista C, Kim IF, Tomashevsky M, Marshall KA, Phillippy KH, Sherman PM, Holko M, et al. NCBI GEO: archive for functional genomics data sets-update. Nucleic Acids Res. 2013;41:D991-5.

50. Mitchell PS, Parkin RK, Kroh EM, Fritz BR, Wyman SK, Pogosova-Agadjanyan EL, Peterson A, Noteboom J, O'Briant KC, Allen A, et al. Circulating microRNAs as stable blood-based markers for cancer detection. Proc Natl Acad Sci U S A. 2008;105:10513-8.

\section{Submit your next manuscript to BioMed Central and take full advantage of:}

- Convenient online submission

- Thorough peer review

- No space constraints or color figure charges

- Immediate publication on acceptance

- Inclusion in PubMed, CAS, Scopus and Google Scholar

- Research which is freely available for redistribution 